LE JOURNAL DE PHYSIQUE ET LE RADIUM.

TOME 15, JANVIER 1954, PAGE 6.

\title{
RÉSONANGE MAGNÉTIQUE SUR DES ATOMES ORIENTÉS OPTIQUEMENT
}

Par Jean BRossel, Bernard CAGNAC et Alfred KASTLER, Laboratoire de Physique, École Normale Supérieure, Paris.

\begin{abstract}
Sommaire. - Des atomes de sodium d'un jet atomique ont été orientés par illumination avec de la lumière de résonance $5890-5896 \AA$ polarisée circulairement. Ces atomes orientés ont été utilisés pour effectuer des transitions de résonance magnétique entre les sous-niveaux Zeeman de l'état fondamental $3^{2} S_{\frac{1}{2}}$, tranisitions qui ont été détectées optiquement. A côté des résonances normales correspondant au passage d'un niveau $m$ à un niveau adjacent on observe, lorsqu'on augmente l'amplitude du champ de haute fréquence, des résonances nouvelles très aigues qui font intervenir à la fois trois ou quatre niveaux Zeeman.
\end{abstract}

La création d'une inégalité de population entre les sous-niveaux Zeeman de l'état fondamental des atomes à l'aide d'un dispositif de " pompage optique " [1] a été obtenue antérieurement [2], [3]. Nous avons perfectionné l'expérience portant sur un jet atomique de sodium. Les taux d'orientation obtenus ont été doublés, et les procédés optiques de détection de l'orientation se sont révélés sensibles et commodes. L'expérience décrite ci-après consiste à prendre un jet d'atomes orientés par le procédé précédent (décrit en détail dans la référence [2]) et à mesurer le signal d'orientation. Puis on provoque une résonance magnétique entre deux sous-niveaux Zeeman de l'état fondamental. 
L'efiet du champ de radiofréquence est d'égaliser la population des deux niveaux impliqués dans la résonance. Le signal d'orientation diminue, ce qui permet la mesure. Le montage utilisé est le suivant :

Un jet atomique de sodium, émis horizontalement, est placé dans un champ magnétique $H_{z}$ perpendiculaire au jet. Celui-ci est illuminé en lumière circulaire droite $\sigma_{1}^{+}\left(\right.$ou gauche $\left.\sigma_{1}^{-}\right)\left({ }^{1}\right)$ sur $23 \mathrm{~cm}$ de long avec la raie de résonance du sodium. La lumière excitatrice se propage dans la direction du champ magnétique $H_{z}$, l'illumination étant simultanée des deux côtés. Ceci constitue la région d'orientation. Le jet passe alors à l'intérieur d'une boucle faisant partie d'un circuit accordé, et dans laquelle on envoie une intensité réglable de radiofréquence. La fréquence utilisée, contrôlée par quartz, est de $108,5 \mathrm{MHz}$. Le champ magnétique oscillant $H_{1}$ est perpendiculaire à $H_{z}$ et en fait, parallèle au jet. C'est dans cette zone de " désorientation " que les transitions sont produites. Le jet aborde alors la région de détection : il y est illuminé en lumière $\pi$. L'intensité circulaire droite $I_{\sigma+}$ réémise est mesurée par un photomultiplicateur qui est monté en opposition avec un deuxième photomultiplicateur mesurant simultanément la lumière circulaire gauche réémise $I_{\sigma-}$.

En l'absence d'orientation des atomes $I_{\sigma+}=I_{\sigma-}$, les deux signaux s'annulent. L'illumination préalable $\sigma_{1}^{+}$détruit la condition précédente et le signal d'orientation $I_{\sigma+}-I_{\sigma-}$ apparaît. Lorsque $H_{\tau}$ varie, la boucle de radiofréquence étant alimentée, on note une baisse du signal d'orientation chaque fois que $H_{3}$ passe par une valeur résonante, c'est-àdire chaque fois que la distance de deux niveaux Zeeman de l'état fondamental du sodium est égale à ı $08,5 \mathrm{MHz}$. C'est ainsi que les courbes des figures I et 2 ont été obtenues : en abcisses, le champ $H_{z}$; en ordonnée sont portées les variations relatives de la quantité

$$
\rho=\frac{I_{\sigma+}-I_{\sigma-}}{I_{\sigma+}+I_{\sigma-}}
$$

Des valeurs de $\rho$ de 35 pour ıoo ont été observées. Pour chaque courbe la puissance de radiofréquence est constante.

La figure I, représente le diagramme Zeeman de l'état fondamental de $\mathrm{Na}$ : deux niveaux hyperfins $F=1$ et $F=2$ distants de I 77 I MHz. Les transitions que nous observons sont les transitions basse fréquence à l'intérieur de chacune des configurations Zeeman de ces deux niveaux. Leurs facteurs de Landé $g_{F}$ sont égaux et de signes opposés $\left( \pm \frac{1}{2}\right) \cdot$ Nous nous plaçons dans une région du champ où l'effet Back-Goudsmit est déjà. sen-

(') Nous dirons que la lumière est circulaire droite lorsque son sens de circulation est celui du courant magnétisant dans les bobines produisant le champ $H_{z}$.
ATOMES ORIENTÉS OPTIQUEMENT

sible : les intervalles entre niveaux contigus sont différents, mais la règle de sélection $\Delta m_{F}= \pm \mathrm{I}$ s'applique encore. On doit donc s'attendre à observer six résonances $A, B_{1}, B_{2}, C_{1}, C_{2}, D$. Mais, il résulte de la formule de Breit-Rabi que les intervalles qui suivent, sont égaux (fig. I) :

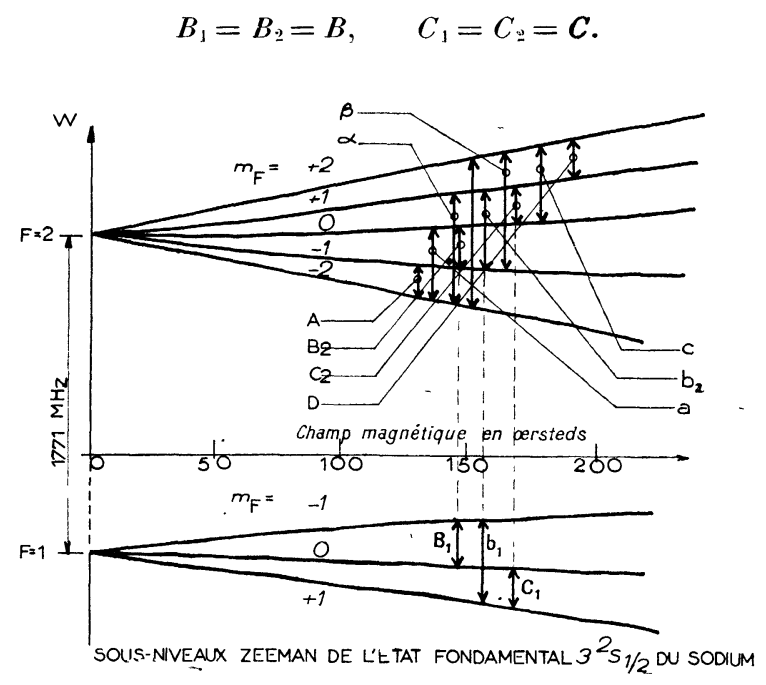

Fig. 1.

Il reste donc en fait quatre résonances distinctes $A$, $B, C, D$. La courbe I (fig. 2) montre qu'avec des puissances de radiofréquence faibles il en est bien ainsi. La résonance $A$, par exemple, correspond à une égalisation, par le champ de radiofréquence, des populations des niveaux $(-2)$ et $(-1)$ de l'état $F=2$, l'illumination préalable en lumière $\sigma_{1}^{-}$ ayant initialement accumulé presque tous les atomes dans le niveau $\left(F, m_{r}\right)=(2,-2)$.

Lorsque l'intensité du champ de radiofréquence croît, un phénomène très remarquable apparaît : la largeur de chacune des résonances $A, B, C, D$ augmente. Lorsqu'elles commencent à se recouvrir (fig. 2, courbe II) trois nouvelles résonances $a, b, c$, apparaissent aux endroits où les distances des niveaux $\left(m_{F} \rightarrow m_{F}^{\prime}\right):(-2 \rightarrow 0),(-1 \rightarrow+1)$ et $(\mathrm{o} \rightarrow+2)$ sont égales à deux fois $108,5 \mathrm{MHz}$. Leur caractère le plus frappant est leur extrême finesse.

Il ne s'agit pas de transitions directes

$$
\Delta m_{F}= \pm 2
$$

(d'ailleurs interdites) provoquées par l'harmonique 2 de l'émetteur de radiofréquence : ces résonances seraient aussi provoquées directement par le fondamental de I08,5 MHz, et cela, dans un champ de 60 gauss environ; rien n'a pu être observé dans cette région.

On doit plutôt admettre que dans les conditions où l'on opère les niveaux $(-2)$ et $(0)$ par exemple peuvent être mis en communication par l'intermédiaire du niveau $(-\mathrm{I})$. La radiofréquence pro- 


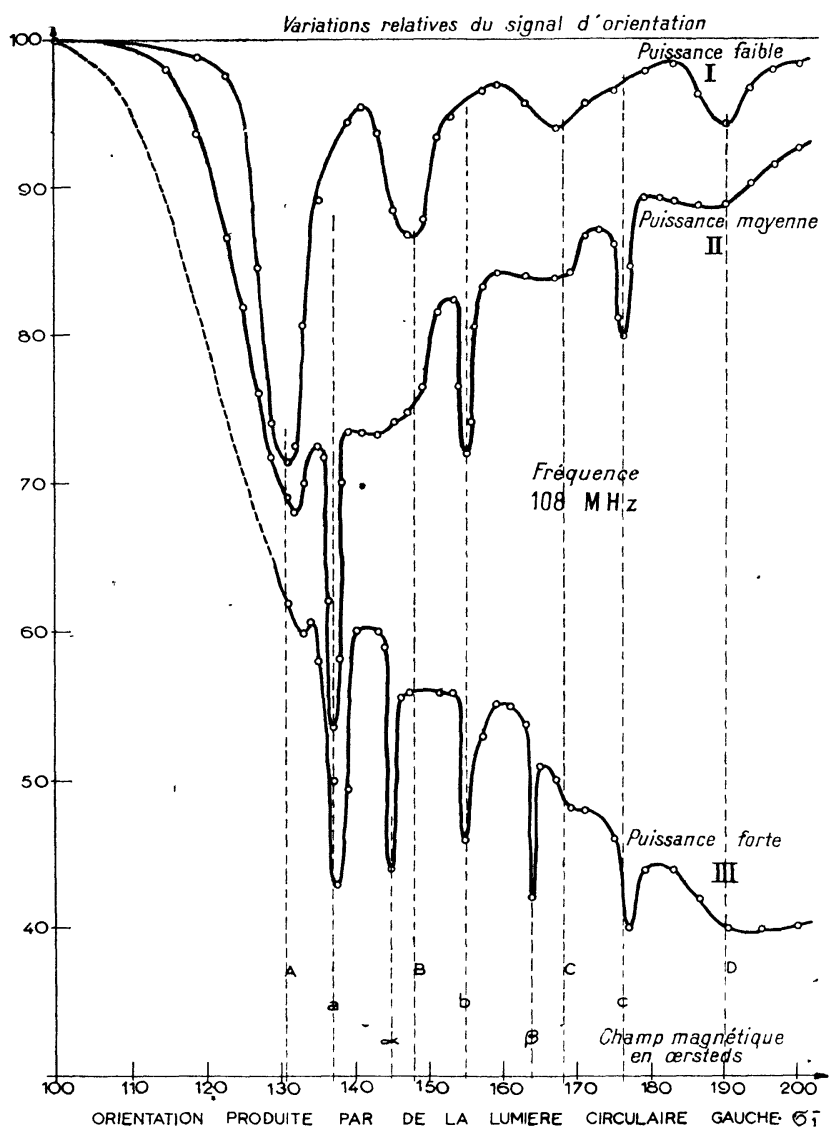

Fig. 2.

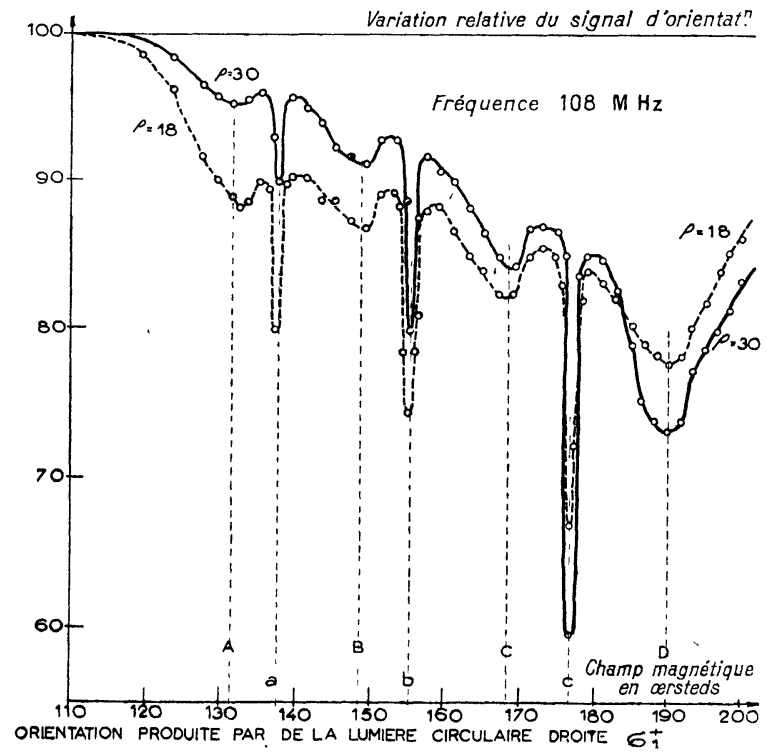

Fig. 3. duit un brassage de population dans lequel les trois niveaux $(-2),(-1)$ et (o) entrent simultanément en jeu. Les populations finales ne sont pas nécessairement égales d'ailleurs. Nous désignerons par convention ces nouvelles résonances par le terme commode de "résonance double " en raison de leur caractère. Leur interprétation quantique sera donnée par ailleurs [4].

Comme prévu, on peut renouveler le processus : en augmentant encore l'intensité de radiofréquence, on provoque à leur tour l'élargissement des résonances doubles et lorsque la zone de recouvrement est suffisante deux " résonances triples " $\alpha$ et $\beta$ apparaissent, correspondant, suivant l'image précédente, à l'intercommunication des niveaux $(-2,-\mathrm{I}, \mathrm{o},+\mathrm{I})$ d'une part et $(-\mathrm{I}, \mathrm{o},+\mathrm{I},+2)$ d'autre part. Ces deux résonances ont été observées aux points où les distances $(-2 \rightarrow+\mathrm{I})$ et $(-\mathrm{I} \rightarrow+2)$ sont égales à trois fois $108,5 \mathrm{MHz}$ (courbe III, fig. 2 ). On peut prévoir aussi une résonance " quadruple ». Nous ne l'avons pas observé avec les puissances de radiofréquence dont nous disposions.

Signalons enfin deux effets apparents sur les figures : on voit sur la figure I que l'intensité de $A$ est plus grande que celle de $B$ :

$$
A>B>C>D, \quad a>b>c .
$$

L'inverse a lieu, si l'orientation préalable, au lieu d'être faite en lumière circulaire gauche $\sigma_{1}^{-}$, l'est en lumière circulaire droite $\sigma_{1}^{+}$(fig. 3). La chose correspond à une accumulation des atomes, dans le premier cas, vers le niveau (-2), dans l'autre, vers le niveau $(+2)$ de l'état $F=2$.

Enfin on note sur la figure 3 l'effet d'une orientation plus ou moins prononcée (lumière $\sigma_{1}^{+}$plus ou moins intense). La courbe en trait plein $\rho=30$ correspond à une orientation forte, avec atomes accumulés en $(+2)$. La raie $A$ a presque disparu, les deux niveaux $(-2)$ et $(-\mathrm{I})$ étant presque complètement vidés et leurs populations étant voisines. La raie $D$ est au contraire très intense [ grande différence de population entre $(+1)$ et $(+2)]$.

La courbe en pointillé $\rho=18$ correspond à une orientation plus faible. Les niveaux $(-2)$ et $(-1)$ sont moins bien vidés : la résonance $A$ est plus forte que dans le cas précédent. Par contre, les niveaux $(+1)$ et $(+2)$ sont moins bien remplis, l'accumulation en $(+2)$ moins prononcée : la résonance $D$ est donc ici plus faible que pour le cas $\rho=30$.

Les expériences décrites ci-dessus doivent permettre une mesure des populations des divers sous-niveaux $m_{F}$ des atomes orientés.

Manuscrit reçu le ro octobre 1953.

BIBLIOGRAPHIE.

[1] Kastler A. - J. Physique Rad., 195o, 11, 255; Physica, I 95 I, 17, 19 I.

[2] Brossel J., Kastler A. et Winter J. - J. Physique Rad., $1952,13,668$.
[3] Hawkins W. B. et Dicke R. H. - Phys. Rev., ig53, 91, r 008 .

[4] Besset C., Horowitz J., Messiah A. M. L., Winter J. - J. Physique Rad., i954, 15, fascicule de février. 